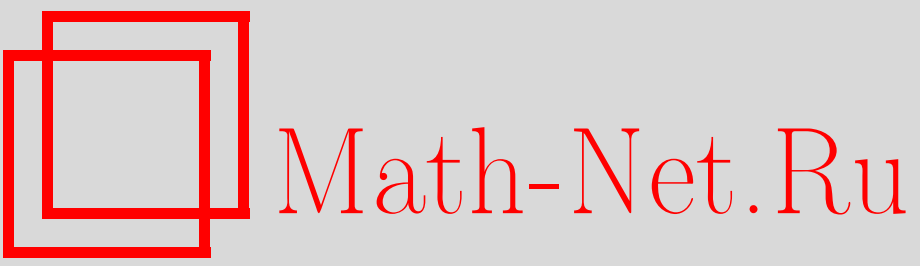

В. И. Субботин, О многогранниках с ромбическими вершинами и правильными гранями, Итоги науки и техн. Сер. Соврем. мат. и ее прил. Темат. обз., 2020, том 181, 112-117

DOI: https://doi.org/10.36535/0233-6723-2020-181-112-117

Использование Общероссийского математического портала Math-Net.Ru подразумевает, что вы прочитали и согласны с пользовательским соглашением

http://www.mathnet.ru/rus/agreement

Параметры загрузки :

IP: 54.162 .127 .20

26 апреля 2023 г., 15:48:22

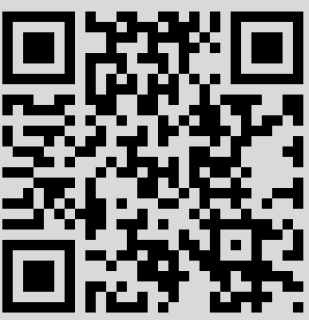




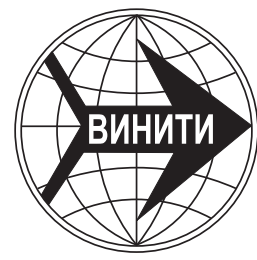

ИТОГИ НАУКИ И ТЕХНИКИ.

Современная математика и ее приложения.

Тематические обзоры.

Том 181 (2020). С. 112-117

DOI: 10.36535/0233-6723-2020-181-112-117

УДК 514.172 .45

\title{
О МНОГОГРАННИКАХ С РОМБИЧЕСКИМИ ВЕРШИНАМИ И ПРАВИЛЬНЫМИ ГРАНЯМИ
}

\author{
(c) 2020 г. $\quad$ В. И. СУББОТИН
}

\begin{abstract}
АннотАция. Рассмотрен класс замкнутых выпуклых многогранников с правильными гранями в $E^{3}$, у которых звезды некоторых вершин симметричны и состоят из равных и одинаково расположенных ромбов $(R R$-многогранники). Доказана теорема о полной классификации $R R$-многогранников с двумя остроугольными ромбическими вершинами, звезды которых отделены поясом правильных граней одного типа. В доказательстве используется ранее полученный автором результат о существовании двух многогранников указанного класса.
\end{abstract}

Ключевые слова: выпуклый многогранник, симметричная ромбическая вершина, звезда вершины, пояс правильных граней, $R R$-многогранник.

\section{ON POLYHEDRA WITH RHOMBIC VERTICES AND REGULAR FACES}

\author{
(c) 2020 V. I. SUBBOTIN
}

\begin{abstract}
In this paper, we consider the class of closed convex polyhedra with regular faces in $E^{3}$ for which the stars of some vertices are symmetric and consist of equal and identically located rhombuses ( $R R$-polyhedra). We obtain a complete classification of $R R$-polyhedra with two acute-angled rhombic vertices whose stars are separated by a belt of regular faces of the same type. The proof is based on a result on the existence of two polyhedra of this class obtained by the author earlier.
\end{abstract}

Keywords and phrases: convex polyhedron, symmetric rhombic vertex, star of the vertex, belt of regular faces, $R R$-polyhedron.

AMS Subject Classification: 52B10, 52B15

В настоящее время существует большое число исследований, посвященных изучению классов многогранников, связанных с правильными многогранниками (см. [1,2,5-10]). Вопрос о возможности комбинаторного или метрического описания всего класса многогранников с заданными условиями симметрии (в частности, условиями правильности) - один из важных вопросов, возникающий при исследовании симметрии многогранников (см. [5]). Если класс многогранников с заданными условиями симметрии содержит класс правильных (платоновых), то в этом случае можно говорить об обобщении класса правильных многогранников. Например, в случае одних лишь условий правильности граней замкнутых выпуклых многогранников в $E^{3}$ найден их полный перечень (многогранники Джонсона-Залгаллера; см. [6, 10]), который, очевидно, содержит в себе правильные многогранники.

Конечно, при описании класса многогранников с заданными условиями симметрии не всегда можно говорить об описании класса с точностью до изометрии или подобия.

Наибольший интерес представляют жесткие условия симметрии, то есть такие условия, которые позволяют описывать класс многогранников по крайней мере с точностью до комбинаторной 
эквивалентности. Например, жесткими являются условия правильности граней для многогранников Джонсона-Залгаллера, а также условие транзитивности группы поворотов (с отражениями) на вершинах многогранника (случай изогонов; см. [5]. Заметим также, что жесткими являются условия симметрии, рассмотренные автором в [3,4]. В частности, в [4] получено полное перечисление так называемых многогранников, сильно симметричных относительно вращения граней («F $S$-многогранников») с точностью до комбинаторного строения и с точностью до сохранения направления и порядка осей вращения каждой грани.

В настоящей работе мы рассмотрим такой тип жестких условий симметрии, который позволит с точностью до подобия описать весь класс многогранников с этими условиями. Условия симметрии приведут к классу многогранников, который содержит, в частности, важный и в кристаллографии многогранник - удлиненный ромбододекаэдр.

Рассмотрим замкнутый выпуклый многогранник $M$ в $E^{3}$. Введем необходимую терминологию. Звезда вершины $V$ многогранника, обозначаемая далее $\operatorname{Star}(V)$ - это совокупность всех граней, имеющих одну общую вершину, совпадающую с $V$. Предположим, что множество $\operatorname{Star}(V)$ состоит из $n$ равных и одинаково расположенных, т.е. сходящихся в вершине $V$ либо своими острыми, либо тупыми углами ромбов (не квадратов). Если $V$ принадлежит оси вращения порядка $n$ множества $\operatorname{Star}(V)$, то в этом случае будем говорить, что $\operatorname{Star}(V)$ - симметричная $n$-ромбическая звезда, а $V$ - симметричная $n$-ромбическая вершина. Если $V$ является вершиной только острых углов ромбов звезды $\operatorname{Star}(V)$, то $V$ будем называть остроугольной. Ромбическую вершину будем называть изолированной, если ее звезда не имеет общих элементов со звездой любой другой ромбической вершины многогранника.

Две равные симметричные $n$-ромбические звезды $\operatorname{Star}(V)$ и $\operatorname{Star}(W)$, расположенные друг к другу своими вогнутыми сторонами, будем называть зеркалъно расположенными, если вершины $V$ и $W$ расположены либо на оси вращения порядка $n$ так, что $\operatorname{Star}(V)$ и $\operatorname{Star}(W)$ симметричны относительно плоскости, либо $\operatorname{Star}(V)$ и $\operatorname{Star}(W)$ могут быть совмещены при помощи зеркального поворота вокруг оси порядка $2 n$, проходящей через их вершины.

Зеркальный поворот вокруг оси $L-$-это композиция поворота вокруг оси $L$ и отражения в плоскости, перпендикулярной этой оси.

Поясом, или замкнутой последовательностью граней, называется связное множество граней $F_{1}, F_{2}, \ldots, F_{n}$ такое, что любые две грани $F_{i}, F_{i+1}$ и также $F_{1}, F_{n}$ имеют только одно общее ребро, а любые две другие грани множества не имеют общих ребер.

Определение 1. Пусть у многогранника существуют симметричные ромбические вершины и существуют грани, не принадлежащие ни одной звезде этих вершин; причем каждая грань, не входящая в звезду ромбической вершины, является правильной. Тогда такой многогранник будем называть $R R$-многогранником (от слов «rombic» и «regular»).

Наша цель в данной работе - найти все $R R$-многогранники с двумя изолированными зеркально расположенными ромбическими звездами, вершины которых симметричные, остроугольные и которые отделены друг от друга поясом правильных граней одного типа. Этот класс многогранников не пуст: ему принадлежит, например, удлиненный ромбический додекаэдр. $R R$-многогранники, удовлетворяющие таким условиям, представляют собой как бы «правильные многогранники с ромбическими вершинами».

Основным результатом работы является следующая теорема.

Теорема 1. Пусть множество ромбических граней $R R$-многогранника $M$ представляет собой две изолированные зеркально расположенные ромбические звезды $\operatorname{Star}(V)$ u $\operatorname{Star}(W)$. Ecли ромбические вершины $V, W$ остроугольные $u$ звезды $\operatorname{Star}(V), \operatorname{Star}(W)$ отделены друг от друга поясом правильных граней одного типа, то $M$ может быть только одним из следующих многогранников:

(i) удлиненным ромбическим додекаэдром;

(ii) 20-гранником, у которого пояс правильных граней составлен из квадратов;

(iii) 24-гранником, у которого пояс правильных граней состоит из треугольников. 
Замечание 1. Многогранники, удовлетворяющие условиям теоремы, называются далее $R R$ многогранниками первого класса.

Доказательство. Пусть $V-n$-ромбическая вершина. Граница ромбической звезды $\operatorname{Star}(V)$ представляет собой замкнутую пространственную ломаную, которую будем обозначать $\Gamma_{V}$. Обозначим $U_{i}, i=1,2, \ldots, n$, вершины границы $\Gamma_{V}$, соседние с вершиной $V$. Пусть $U$ - одна из этих вершин. Обозначим $\alpha$ угол с вершиной $U$, сторонами которого являются две стороны соседних ромбов, принадлежащих $\Gamma_{V}$. Угол $\alpha$ и все ему эквивалентные в $\Gamma_{V}$ для наглядности будем называть свободными.

Отметим, что если стороны каждого угла $\alpha_{i}, i=1,2, \ldots, n$, с вершиной $U_{i}$ являются двумя сторонами соответственно только одного равностороннего треугольника $T_{i}$, то мы не получим пояса из треугольных граней, отделяющего две ромбические звезды $\operatorname{Star}(V)$ и $\operatorname{Star}(W)$. Действительно, в этом случае множество граней $T_{i}, i=1,2, \ldots, n$, и всех соседних с ними треугольных граней не будет удовлетворять определению пояса (рис. 1(а)). Таким образом, при любом $n \geqslant 3$ в свободные углы $\alpha_{i}, i=1,2, \ldots, n$, звезды $\operatorname{Star}(V)$ нельзя поместить только по одному равностороннему треугольнику.

1. Рассмотрим случай, когда $n=3$, то есть случай трехгранной симметричной ромбической вершины. Покажем, что в этом случае не существует многогранников, удовлетворяющих условиям теоремы.

В свободные углы нельзя поместить по два равносторонних треугольника. Действительно, в случае существования у такого многогранника, очевидно, возможна только зеркально-поворотная ось $V W$. Однако пояс треугольных граней $A B C D E F \ldots$ (рис. $1(\mathrm{~b})$ ) в этом случае невозможен. Действительно, вместо граней $A B$ и $E F$ мы получим, соответственно, два параллелограмма $P$ и $Q$ (рис. 1(c)) и две ромбические вершины многогранника будут отделены поясом равных параллелограммов (см. [3]). Это так называемый удлиненный ромбоэдр. Он является по терминологии [3] одним из $R S$-многогранников.

Так как внутренние острые углы ромбов 3-ромбической симметричной вершины $V$ равны свободным углам (рис. $1(\mathrm{~d})$ ), то при $n=3$ нельзя поместить в свободные углы правильный $k$ угольник при $k>3$. Поэтому в случае $n=3$ не существует $R R$-многогранников первого класса.

2. Рассмотрим теперь случай $n=4$. Покажем, что в этом случае существует только два многогранника, удовлетворяющих условиям теоремы.

Невозможны многогранники с помещенными в свободные углы по одному равностороннему треугольнику. Это показывается так же, как и в случае $n=3$.

Если в свободные углы поместить по два равносторонних треугольника, то существует 24-гранник с зеркально-поворотной осью $V W$ 8-го порядка (рис. 2(c)). Существование и единственность такого многогранника доказаны автором в [3].

Покажем, что при $n=4$ не существует многогранника с помещенными в свободные углы квадратами.

Для этого рассмотрим два смежных ребра $m$ и $n$, инцидентные вершине $S$ (рис. 1(e)), которые являются сторонами квадратной грани. Переходя от пары ребер $(m, n)$ к паре ребер $(p, q)$, где $m\|p, n\| q$, затем к паре $(r, t)$, где $r\|p, t\| q$, получим, что острый угол ромба равен $\pi / 2$. Это противоречит тому, что ромбические вершины остроугольные. Полученное противоречие доказывает, что в этом случае многогранник невозможен.

Невозможность многогранника при $n=4$ с поясом из правильных $k$-угольников, $k \geqslant 6$, для случая зеркально-поворотной оси вращения, следует из того, что в вершине $S$ и ей эквивалентных (рис. $1(\mathrm{e})$ ), в этом случае сумма плоских углов будет больше $2 \pi$.

При $n=4$ пояс из правильных 5-угольников, отделяющий две ромбические звезды $\operatorname{Star}(V)$ и $\operatorname{Star}(W)$, построить нельзя и в случае обычной оси вращения, и в случае зеркально-поворотной оси (рис. 1(f), (g)). То же верно, очевидно, и для правильных $k$-угольников для $k=7$.

В случае $k>7$ невозможен пояс из правильных $k$-угольников и в случае обычной оси вращения, так как в этом случае сумма плоских углов в вершине $U$ и ей эквивалентных будет больше $2 \pi$. 


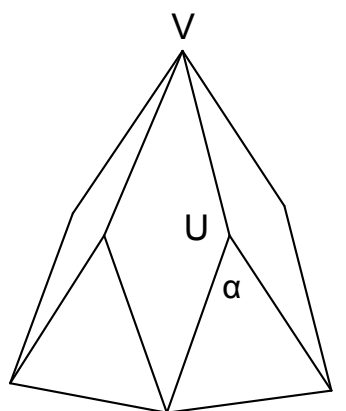

a)

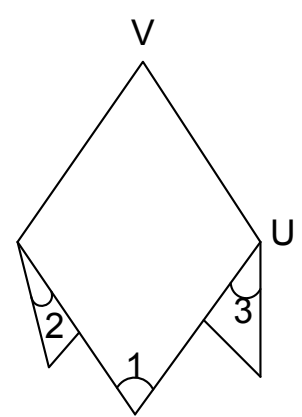

d)

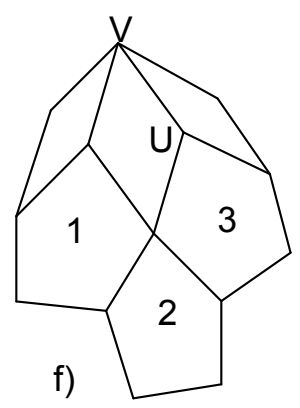

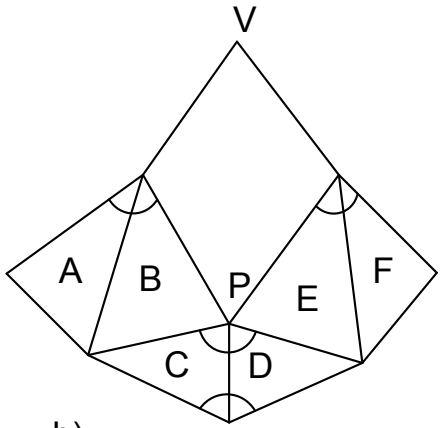

b)

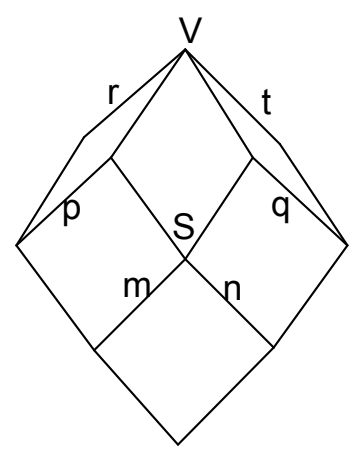

e)

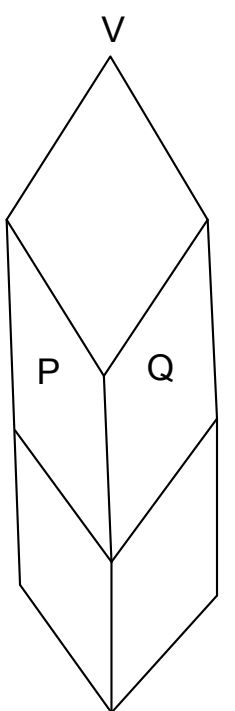

c)

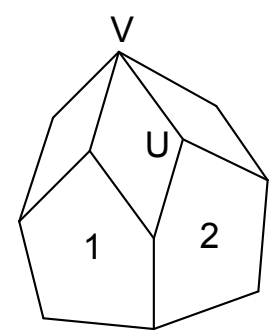

g)

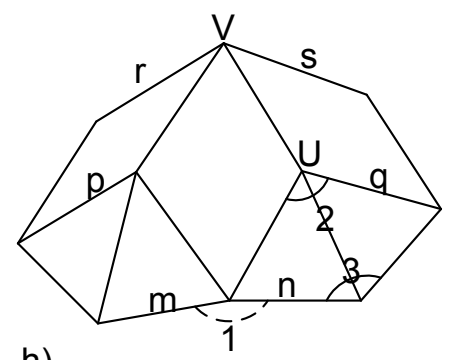

h)

Рис. 1. К доказательству теоремы 1

Таким образом, в случае $n=4$ остается возможность существования многогранника с поясом правильных 6-угольников и с обычной осью вращения 4-го порядка. Такой многогранник действительно существует. Это известный удлиненный ромбический додекаэдр (рис. 2(a)). По терминологии [4] этот многогранник является одним из полностью перечисленных в [4] $R S$-многогранников.

3. $n=5$. Пусть к свободным углам $\alpha_{i}$ приставлены по два равносторонних треугольника. Тогда угол 1 между ребрами $m$ и $n$ не равен углу между ребрами $p$ и $q$ (рис. $1(\mathrm{~h})$ ). Действительно, в этом случае пары $(m, p),(n, q)$ не являются противоположными сторонами параллелограммов. Переходя по параллельным ребрам от угла между ребрами $p$ и $q$ к углу между ребрами $r$ и $s$ и т. д., получим, что угол 1 не равен ни одному из углов $\alpha_{i}$, в частности, не равен углу 2 на рис. 1 . Но угол 2 равен углу 3 , а угол 3 эквивалентен углу 1 при зеркально-поворотной симметрии многогранника.

Полученное противоречие доказывает невозможность многогранника в этом случае.

Если при $n=5$ к углам $\alpha_{i}$ приставлены квадраты, то возможна только зеркально-поворотная ось вращения 10-го порядка многогранника. Такой 20-многогранник существует: у него 5- 


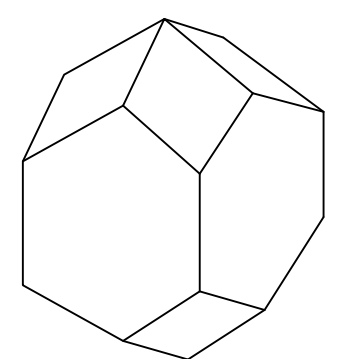

a)

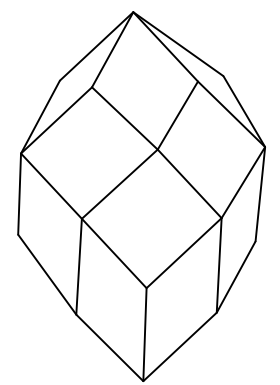

b)

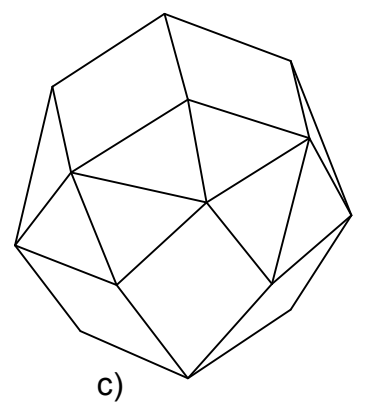

Рис. 2. RR-многогранники

ромбические вершины отделены поясом из десяти квадратов (рис. 2(b)). Существование и единственность этого многогранника доказано автором в [3].

Другие случаи при $n=5$ рассматриваются аналогично случаю $n=4$.

4. Рассмотрим случай, когда $n \geqslant 6$. Покажем, что в этом случае не существует многогранников, удовлетворяющих условиям теоремы.

Если стороны каждого угла $\alpha_{i}$ являются сторонами двух равносторонних треугольников, то в каждой вершине $U_{i}$ сумма плоских углов будет больше $2 \pi$, так как тупые внутренние углы ромбов звезды при $n \geqslant 6$ будут больше $2 \pi / 3$ (рис. $1(\mathrm{~h})$ ).

Покажем теперь, что если к углам $\alpha_{i}$ приставлены квадраты, то такой многогранник тоже не может существовать при $n \geqslant 6$. Действительно, если $n>7$, то в вершинах $U_{i}$ сумма плоских углов будет, как и в случае двух треугольных граней, больше $2 \pi$.

Если $n=6,7$, то и в этом случае многогранник невозможен. Действительно, предположим, что такой многогранник с поясом из квадратов существует.

При $n=6$ рассмотрим трехгранный угол с вершиной $U$. Рассмотрим два смежных ребра $m$ и $n$, инцидентные вершине $U$, которые являются сторонами квадратной грани и сторонами смежных ромбов. Две стороны $p$ и $q$ ромбов звезды $\operatorname{Star}(V)$, соответственно параллельные ребрам $m$ и $n$, перпендикулярны друг другу (рис. 1(e)).

Аналогично перейдем к паре взаимно перпендикулярных ребер $r$ и $s$, где $r\|p, s\| q$, принадлежащих $\operatorname{Star}(V)$. Продолжая этот переход, мы получим пару ребер, которые должны быть перпендикулярны, но оказываются на завершающем шаге этого перехода сторонами острого угла ромба звезды $\operatorname{Star}(V)$. Полученное противоречие доказывает, что при $n=6$ многогранник с поясом квадратов существовать не может.

Аналогично проведем доказательство для случая 7-ромбической вершины $V$. В этом случае на завершающем шаге перехода по параллельным ребрам мы получим вместо взаимно перпендикулярных ребер два параллельных ребра.

Если к углам $\alpha_{i}$ приставлены правильные пятиугольники (рис. $\left.1(\mathrm{f}),(\mathrm{g})\right)$, то и такой многогранник не может существовать при $n \geqslant 6$, как в случае зеркально-поворотной оси, так и в случае обычной оси вращения порядка $n$. Действительно, в этом случае мы не получим пояса из правильных пятиугольников, отделяющего две ромбические вершины $V, W$.

В случае $n \geqslant 6$ к углам $\alpha_{i}$ нельзя приставить правильные $k$-угольники при $k \geqslant 6$, так как в каждой вершине $U_{i}$ сумма плоских углов будет больше $2 \pi$. Действительно, в этом случае каждый острый угол при $n$-ромбической вершине $V$ будет меньше $\pi / 3$, а следовательно, каждый тупой угол ромбов звезды $\operatorname{Star}(V)$ будет больше $2 \pi / 3$. Теорема доказана.

\section{СПИСОК ЛИТЕРАТУРЫ}

1. Деза М., Гришухин В. П., Штогрин А. И. Изометрические полиэдральные подграфы в гиперкубах и кубических решетках. - М.: МЦНМО, 2007. 
2. Залгаллер В. А. Выпуклые многогранники с правильными гранями// Зап. науч. семин. ЛОМИ. 1967. - 2. - C. 1-220.

3. Субботин В. И. О двух классах многогранников с ромбическими вершинами// Зап. науч. семин. ПОМИ. - 2018. - 476. - С. 153-164.

4. Субботин В. И. Об одном классе сильно симметричных многогранников// Чебышев. сб. - 2016. № 4. - С. $132-140$.

5. Coxeter H. S. M. Regular Polytopes. - New York: Dover, 1973.

6. Coxeter H. S. M. Regular and semi-regular polytopes. III// Math. Z. - 1988. — 200, № 21. — P. 3-45.

7. Cromwell P. R. Polyhedra. - Cambridge: Cambridge Univ. Press, 1999.

8. Farris S. L. Completely classifying all vertex-transitive and edge-transitive polyhedra// Geom. Dedic. 1988. - 26, № 1. - P. 111-124.

9. Grunbaum B. Regular polyhedra - old and new// Aequat. Math. - 1977. — 16, № 1-2. - P. 1-20.

10. Johnson N. W. Convex polyhedra with regular faces// Can. J. Math. — 1966. — 18, № 1. — P. 169-200.

Субботин Владимир Иванович

Южно-Российский государственный политехнический университет (Новочеркасский политехнический институт) им. М. И. Платова, Новочеркасск

E-mail: geometry@mail.ru 